\title{
STUDY OF ANTHROPOMETRIC MEASUREMENTS, BIOCHEMICAL PARAMETERS AND HORMONAL LEVELS IN WOMEN WITH PCOS AT A TERTIARY CENTER OF RURAL HARYANA
}

\author{
Poonam Taneja1 , Bindoo Yadav², Ashok Kumar Shah³, Vinita Gupta4, Pawan Tiwari ${ }^{5}$
}

${ }^{1}$ Associate Professor, Department of Obstetrics and Gynaecology, SGT Medical College, Hospital and Research Institute, Gurugram, Haryana, India.

${ }^{2}$ Associate Professor, Department of Obstetrics and Gynaecology, SGT Medical College, Hospital and Research Institute, Gurugram, Haryana, India.

${ }^{3}$ Assistant Professor, Department of Obstetrics and Gynaecology, WCMS (World College of Medical Sciences and Research), Jhajjar, Haryana, India.

${ }^{4}$ Professor, Department of Obstetrics and Gynaecology, SGT Medical College, Hospital and Research Institute, Gurugram, Haryana, India.

5Professor, Department of Obstetrics and Gynaecology, SGT Medical College, Hospital and Research Institute, Gurugram, Haryana, India.

\section{ABSTRACT}

\section{BACKGROUND}

During the past decade, our understanding of pathophysiology of PCOS has undergone a remarkable evolution. Despite our familiarity with this disease, some of its fundamental characteristics remains poorly recognized and understood. The present study focuses on comprehensive knowledge about the current and emerging concepts of the PCOS such as, pathogenesis, diagnosis and better management of the syndrome. Obesity was found to be one of the important factors that contribute to the development of long-term risk of PCOS. In addition, increased obesity and abdominal adiposity further aggravate the clinical, hormonal and metabolic parameters in PCOS and, if treated, can reverse most of these abnormalities to a clinically significant degree. Our study suggests that anthropometric parameters (especially BMI \& WHR) are significantly correlated with lipid profile in PCOS patients confirming the importance of early treatment of obesity to prevent further complications in the future. We wanted to study the anthropometric measurements, the hormonal status and biochemical parameters in females diagnosed with PCOS, study the correlation between them, and compare them with controls.

\section{METHODS}

In this prospective case control study, women clinically diagnosed with PCOS $(\mathrm{N}=100)$ and age matched healthy control female subjects $(\mathrm{N}=100)$ in the age group (18-35) years were enrolled. Body mass index (BMI), waist to hip ratio (WHR), fasting blood glucose, lipid profile and hormonal levels were estimated in both groups and further compared using student $t$-test. Anthropometric measurements were also correlated with the hormonal levels \& biochemical parameters and the results were analysed using Pearson's correlation coefficients. Sample size was taken for convenience.

\section{RESULTS}

We found that the levels of TSH, LH, FSH, LH/FSH ratio, prolactin, TC, TG, LDL \& TC/HDL ratio were significantly raised in PCOS females as compared to healthy females $(\mathrm{p}<0.0001)$. Anthropometric measurements (BMI, waist-to-hip ratio) showed significant positive correlation with TSH, biochemical parameters (TC, TG, LDL \& TC/HDL ratio) and hormonal profile (LH, FSH, LH/FSH ratio \& Prolactin) of PCOS women.

\section{CONCLUSIONS}

There is significant derangement in hormonal \& biochemical status of women suffering from PCOS that leads to an altered energy metabolism and endocrinological cascade of PCOS. Also, the anthropometric measurements were statistically significantly different from controls which showed significant positive correlation with the hormonal and biochemical parameters. This emphasizes the importance of early treatment of obesity, timely identification of hypothyroidism and biochemical derangement to prevent complications in the future. Awareness, Prevention, and Treatment, of PCOS at an early stage should be actively taken up for a healthy woman and a healthy nation.

HOW TO CITE THIS ARTICLE: Taneja P, Yadav B, Shah AK, et al. Study of anthropometric measurements, biochemical parameters and hormonal levels in women with PCOS at a tertiary center of rural Haryana. J. Evolution Med. Dent. Sci. 2019;8(16):1311-1317, DOI: $10.14260 /$ jemds/2019/292

\section{BACKGROUND}

Polycystic ovary syndrome is a common endocrinological disorder amongst women of reproductive age, yet the root

'Financial or Other Competing Interest': None.

Submission 23-02-2019, Peer Review 10-04-2019,

Acceptance 17-04-2019, Published 22-04-2019.

Corresponding Author:

Dr. Bindoo Yadav,

641/16, Ovil Lines, Gurugram-122001, Haryana, India.

E-mail:abry90@yahoo.co.in

DOI: $10.14260 /$ jemds $/ 2019 / 292$

\section{(c) $(1)(-$}

cause is still to be evaluated ${ }^{1}$. It is known to be associated with the abnormality of hypothalamic-pituitary -ovarian axis and the abnormal feedback mechanism by ovarian oestrogen resulting in relative increase of $\mathrm{LH}$ to $\mathrm{FSH}$ release ratio. Environmental factors implicated in PCOS (e.g., obesity) can be exacerbated by poor dietary choices and physical inactivity; infectious agents and toxins may also play a role. ${ }^{2}$ The reproductive and metabolic features of PCOS are sometimes reversible with lifestyle modifications such as weight loss and exercise. ${ }^{3}$ Approximately $50 \%$ of PCOS women are overweight or obese which may play a pathogenic role in the development of insulin resistance and associated 
hyperinsulinemia in women with PCOS.4-6 Prevalence of obesity and diabetes mellitus in most industrialized countries including India is also on the rise owing to urbanization and change in lifestyle. Recently a few studies among adolescents in schools report prevalence of PCOS as $9.13 \%$ to $36 \% .7,8$ Gainie and Kalra ${ }^{9}$ have pointed out that the cost of handling the associated multiple consequences of PCOS will be a huge drain on the overburdened health resources of India. It is time to acknowledge PCOS as an important noncommunicable disease. ${ }^{10}$ Moreover the cost involved in diagnosis accounts only for a fraction of the total costs of managing PCOS (approximately 2\%). Screening for the disorder will bring about early intervention and possibly prevention of serious sequel. ${ }^{11}$ In view of the above this study was conducted to study the association of anthropometric measurements, biochemical parameters and hormonal levels in women with PCOS and to determine the significance of these levels in low resource centers.

\section{METHODS}

In this prospective, case control study, women clinically diagnosed with PCOS $(\mathrm{N}=100)$ and age matched healthy control female subjects $(\mathrm{N}=100)$ in the age group (18-35) years were enrolled \& conducted in the department of Obstetrics and Gynecology; SGT medical college, Hospital and Research Institute (SGT University) Gurugram-Delhi-NCR, India. The study was approved by Institutional Ethical Committee. An informed written consent was obtained from all subjects at the study entry after apprising them the nature and objectives of the study. Sample size was taken for convenience.

Selection of cases and controls was done by simple random sampling.

The study group comprised two hundred Indian women (Aged 15 to 35 years) from 2014 to 2017. One hundred patients with a clinical diagnosis of polycystic ovary syndrome (PCOS) were recruited for the study from the outpatient department (OPD) of Obstetrics and Gynaecology of SGT medical college, Hospital and Research Institute. One hundred healthy age matched subjects were randomly selected from the students and staff of SGT Medical College and recruited as controls.

The diagnosis of PCOS of all suspected cases was made according to the revised criteria of the Rotterdam ESHRE/ASRM-sponsored PCOS Consensus Workshop group based on their symptoms, Investigations and was further confirmed by high resolution ultra-solography. As per "Rotterdam revised criteria"12-13 which requires the presence of at least two out of three features i.e., Oligo- and/or anovulation, clinical and/or biochemical evidence of hyperandrogenism and or ultra-sonographic findings of polycystic ovaries. The controls were age matched females who did not have any menstrual irregularity, hirsutism, or signs of hyperandrogenism.

The study groups were screened based on Rotterdam revised criteria.

\section{Study Design}

Prospective case control study.

\section{Place and Duration of Study}

Sri Guru Gobind Singh Tricentenary Hospital, Medical College and Research Institute, SGT University, Budhera, Gurugram, Haryana between 2014 to 2017.

\section{Inclusion Criteria}

All PCOS women as per the Rotterdam revised criteria.

\section{Exclusion Criteria}

The subjects with hypertension, syndrome of severe insulin resistance, diabetes mellitus, cardiovascular disease, congenital adrenal hyperplasia, androgen secreting tumours, Cushing syndrome, history of smoking and/or tobacco intake, androgenic/anabolic drug use or abuse or thyroid dysfunction and hyperprolactinemia were excluded from the study

\section{Statistical Methods}

All the parameters studied were expressed as Mean and standard deviation. Statistical analyses were performed using the Microsoft excel sheets, SPSS version 24.0 for windows. To determine the difference between mean levels of the cases and the controls, independent sample t-test was used. Pearson's correlation analysis was employed to analyse data and to elucidate any relationship between the anthropometry, biochemical and hormonal parameters in PCOS women.

\section{RESULTS}

The present study was done to correlate anthropometry, biochemical status and hormonal levels amongst women with PCOS controls. Data was analysed for differences in anthropometric parameters, lipid profile, hormonal levels and serum biochemical markers between PCOS patients and healthy controls were studied by independent (Unpaired) Student's t-test.

Association of anthropometric, biochemical and hormonal parameters in PCOS patients were studied by Pearson's correlation analysis.

\section{Anthropometric Measurements}

The women diagnosed with PCOS $(n=100)$ and the healthy controls $(n=100)$ were in the age group (18-35 years). The mean age of PCOS patients was $(23.34 \pm 4.33)$ years and for controls was $(22.44 \pm 2.43)$ years Anthropometric measurements (BMI: body mass index, WHR: waist to hip ratio) of PCOS patients and healthy subjects is presented in Table A

\begin{tabular}{|c|c|c|c|c|}
\hline Variables & PCOS Cases & Control & t-Value & p-Value \\
\hline Age (Years $)$ & $23.34 \pm 4.33$ & $22.44 \pm 2.43$ & 1.81 & 0.07 \\
\hline $\begin{array}{c}\text { BMI } \\
\left(\left(\mathrm{Kg} / \mathrm{m}^{2}\right)\right.\end{array}$ & $25.21 \pm 3.34$ & $20.32 \pm 2.63$ & 11.48 & $<0.001^{* *}$ \\
\hline WHR & $0.86 \pm .05$ & $0.77 \pm 0.05$ & 11.33 & $<0.001^{* *}$ \\
\hline \multicolumn{5}{|c|}{ Table A } \\
\hline
\end{tabular}

BMI and WHR were significantly increased in PCOS patients compared to healthy controls.

\begin{tabular}{|c|c|c|c|c|}
\hline Variables & $\begin{array}{c}\text { PCOS } \\
\text { Cases }\end{array}$ & Controls & t-Value & p-Value \\
\hline $\begin{array}{c}\text { Fasting Blood } \\
\text { Sugar } \\
\text { (mg/dL) (FBS) }\end{array}$ & $\begin{array}{c}92.76 \pm \\
8.21\end{array}$ & $\begin{array}{c}89.43 \pm \\
7.39\end{array}$ & 3.01 & $0.003^{* *}$ \\
\hline $\begin{array}{c}\text { Total Cholesterol } \\
\text { (mg/dL) )(TC) }\end{array}$ & $\begin{array}{c}172.52 \pm \\
31.16\end{array}$ & $\begin{array}{c}142.22 \pm \\
15.57\end{array}$ & 8.69 & $<0.001^{* *}$ \\
\hline $\begin{array}{c}\text { Triglycerides } \\
\text { (mg/dL) (TG) }\end{array}$ & $\begin{array}{c}128.4 \pm \\
48.54\end{array}$ & $\begin{array}{c}91.82 \pm \\
21.79\end{array}$ & 6.87 & $<0.001^{* *}$ \\
\hline $\begin{array}{c}\text { High Density } \\
\text { Lipoproteins- }\end{array}$ & $\begin{array}{c}41.72 \pm \\
5.32\end{array}$ & $\begin{array}{c}46.89 \pm \\
3.86\end{array}$ & -7.85 & $<0.001^{* *}$ \\
\hline
\end{tabular}




\begin{tabular}{|c|c|c|c|c|}
\hline $\begin{array}{c}\text { Cholesterol } \\
(\mathrm{mg} / \mathrm{dL}))(\mathrm{HDL})\end{array}$ & & & & \\
\hline $\begin{array}{l}\text { Low Density } \\
\text { Lipoproteins- } \\
\text { Cholesterol } \\
(\mathrm{mg} / \mathrm{dL}) \text { ) (LDL) }\end{array}$ & $\begin{array}{c}116.65 \pm \\
28.5\end{array}$ & $\begin{array}{c}84.18 \pm \\
12.48\end{array}$ & 10.43 & $<0.001^{* *}$ \\
\hline $\begin{array}{l}\text { Very Low-Density } \\
\text { Lipoproteins- } \\
\text { Cholesterol } \\
\text { VLDL-C }(\mathrm{mg} / \mathrm{dL}) \\
\end{array}$ & $\begin{array}{c}25.68 \pm \\
9.7\end{array}$ & $\begin{array}{c}18.36 \pm \\
4.35\end{array}$ & 6.87 & $<0.001^{* *}$ \\
\hline $\begin{array}{c}\text { LDL-C/HLDL-C } \\
\text { Ratio } \\
\end{array}$ & $\begin{array}{c}2.85 \pm \\
0.86 \\
\end{array}$ & \begin{tabular}{|l|}
$1.8 \pm$ \\
0.29 \\
\end{tabular} & 11.5 & $<0.001^{* *}$ \\
\hline TC/ HDL-C Ratio & $4.2 \pm 0.95$ & $3.05 \pm 0.41$ & 11.06 & $<0.001^{* *}$ \\
\hline \multicolumn{5}{|c|}{ Table B } \\
\hline
\end{tabular}

\section{Biochemical Parameters}

Biochemical parameters of PCOS patients and healthy subjects is presented in Table B. Fasting blood sugar (FBS) in PCOS patients $(\mathrm{p}=0.003)$ compared to healthy subjects was significantly raised. All lipids studied such as total cholesterol (TC), tri glycerides (TG), low density lipoprotein cholesterol (LDL-C) and very low density lipoprotein cholesterol (VLDLC) were significantly increased in PCOS patients compared to control group $(\mathrm{p}<0.001)$ whereas high density lipoprotein cholesterol (HDL-C) was significantly decreased in PCOS patients compared to control group $(\mathrm{p}<0.001)$ and the lipid risk factors such as LDL-C/HDL-C ratio and TC/HDL-C ratio were significantly elevated in PCOS patients $(\mathrm{p}<0.001)$ ** compared to controls.

\section{Hormonal levels}

Table C shows the hormonal levels of PCOS patients and healthy subjects. We found significant elevation in hormonal parameters such as thyroid stimulating hormone (TSH); luteinizing hormone (LH); follicle stimulating hormone (FSH); prolactin (PRL), and LH/LSH ratio in PCOS patients $(\mathrm{p}<0.001)$ compared to controls.

\begin{tabular}{|c|c|c|c|c|}
\hline Variables & PCOS cases & Controls & t-value & p-value \\
\hline TSH $(\mu \mathrm{IU} / \mathrm{dL})$ & $3.39 \pm 2.82$ & $1.67 \pm 0.86$ & 5.8 & $<0.001^{* *}$ \\
\hline LH $(\mathrm{mIU} / \mathrm{L})$ & $8.2 \pm 6.22$ & $3.6 \pm 0.93$ & 7.32 & $<0.001^{* *}$ \\
\hline FSH $(\mathrm{mIU} / \mathrm{L})$ & $5.71 \pm 2.38$ & $4.45 \pm 1.05$ & 4.81 & $<0.001^{* *}$ \\
\hline PRL $(\mathrm{ng} / \mathrm{dL})$ & $13.31 \pm 8.4$ & $7.39 \pm 1.58$ & 6.92 & $<0.001^{* *}$ \\
\hline LH/FSH ratio & $1.67 \pm 1.23$ & $0.81 \pm 0.14$ & 6.92 & $<0.001^{* *}$ \\
\hline \multicolumn{5}{|c|}{ Table C } \\
\hline
\end{tabular}

\section{Correlation Analysis}

\section{Association of Anthropometric Measurements and Lipid Profile}

Analysis of test was done by an independent (Unpaired) student's test. $\mathrm{p}<.001^{* *}$ when compared with control subjects. The present study showed significant Correlation between anthropometric measurements and lipid profile in PCOS patients (Table D).

\begin{tabular}{|c|c|c|c|c|}
\hline Variables & \multicolumn{2}{|c|}{ BMI } & \multicolumn{2}{c|}{ WHR } \\
\hline & $\mathrm{r}$ & $\mathrm{p}$ & $\mathrm{r}$ & $\mathrm{p}$ \\
\hline TC & 0.50 & $0.001^{* *}$ & 0.42 & $0.001^{* *}$ \\
\hline TG & 0.44 & $0.001^{* *}$ & 0.40 & $0.001^{* *}$ \\
\hline HDL-C & -0.25 & $0.001^{* *}$ & -0.32 & $0.001^{* *}$ \\
\hline LDL-C & 0.57 & $0.001^{* *}$ & 0.44 & $0.001^{* *}$ \\
\hline VLDL-C & 0.44 & $0.001^{* *}$ & 0.40 & $0.001^{* *}$ \\
\hline $\begin{array}{c}\text { LDL-C/ HDL-C } \\
\text { Ratio }\end{array}$ & 0.54 & $0.001^{* *}$ & 0.47 & $0.001^{* *}$ \\
\hline TC/ HDL-C Ratio & 0.50 & $0.001^{* *}$ & 0.48 & $0.001^{* *}$ \\
\hline \multicolumn{5}{|c|}{ Table D } \\
\hline
\end{tabular}

BMI had a significant positive relationship with TC, TG, LDLC, VLDL-C, LDL-C/HDL-C ratio, and TC/HDL-C ratio respectively and significant negative co-relation with HDL-C whereas, WHR showed positive correlation with TC, TG, LDL, VLDL-C, LDL-C/HDL-C ratio and TC/HDL ratio and significant negative correlation with HDL-C PCOS patients.

\section{Association of Anthropometric Measurements and Hormonal Profile (Table E)}

Analysis of data was done by Pearson's co-relation. $\mathrm{r}$ (Corelation coefficient) (Statistically significant at $\mathrm{p}<0.05$ )*, (Statistically significant at $\mathrm{p}<0.01$ )** When compared with control subjects.

\begin{tabular}{|c|c|c|c|c|}
\hline Variables & \multicolumn{2}{|c|}{ BMI } & \multicolumn{2}{c|}{ WHR } \\
\hline & $\mathrm{r}$ & $\mathrm{p}$ & $\mathrm{r}$ & $\mathrm{p}$ \\
\hline TSH & 0.31 & $0.001^{* *}$ & 0.35 & $0.001^{* *}$ \\
\hline LH & 0.15 & $0.02^{*}$ & 0.28 & $0.001^{* *}$ \\
\hline FSH & 0.20 & $0.004^{* *}$ & 0.29 & $0.001^{* *}$ \\
\hline PRL & 0.24 & $0.001^{* *}$ & 0.31 & $0.001^{* *}$ \\
\hline LH/FSH Ratio & 0.14 & $0.04^{*}$ & 0.25 & $0.001^{* *}$ \\
\hline \multicolumn{5}{|c|}{ Table E } \\
\hline
\end{tabular}

In the present study, hormonal parameters showed significant positive correlation between anthropometric measurements such as BMI and WHR in PCOS patients BMI had a significant positive relationship with TSH, LH, FSH, PRL, and LH/FSH ratio respectively whereas, WHR showed positive correlation with TSH, LH, FSH, PRL, and LH/FSH ratio in PCOS patients.

\begin{tabular}{|c|c|c|c|c|c|c|}
\hline Variables & & TSH & LH & FSH & PRL & $\begin{array}{c}\text { LH/FSH } \\
\text { ratio }\end{array}$ \\
\hline \multirow{2}{*}{ TC } & $\mathrm{r}$ & 0.19 & 0.25 & 0.22 & 0.10 & 0.17 \\
\cline { 2 - 7 } & $\mathrm{p}$ & $0.006^{* *}$ & $0.001^{* *}$ & $0.001^{* *}$ & 0.14 & $0.01^{*}$ \\
\hline \multirow{2}{*}{$\mathrm{TG}$} & $\mathrm{r}$ & 0.33 & 0.08 & 0.23 & 0.11 & 0.01 \\
\cline { 2 - 7 } & $\mathrm{p}$ & $0.001^{* *}$ & 0.23 & $0.001^{* *}$ & 0.10 & 0.84 \\
\hline \multirow{2}{*}{ HLDL-C } & $\mathrm{r}$ & -0.16 & -0.19 & -0.15 & -0.22 & -0.18 \\
\cline { 2 - 7 } & $\mathrm{p}$ & $0.02^{*}$ & $0.007^{* *}$ & $0.02^{*}$ & $0.002^{* *}$ & $0.008^{* *}$ \\
\hline \multirow{2}{*}{ LDL-C } & $\mathrm{r}$ & 0.24 & 0.13 & 0.22 & 0.18 & 0.09 \\
\cline { 2 - 7 } & $\mathrm{p}$ & $0.001^{* *}$ & $0.04^{*}$ & $0.001^{* *}$ & $0.008^{* *}$ & 0.18 \\
\hline \multirow{2}{*}{ VLDL-C } & $\mathrm{r}$ & 0.33 & 0.08 & 0.23 & 0.11 & 0.01 \\
\cline { 2 - 7 } & $\mathrm{p}$ & $0.001^{* *}$ & 0.23 & $0.001^{* *}$ & 0.10 & 0.84 \\
\hline $\begin{array}{c}\text { LDL- } \\
\text { C/HLDL-C } \\
\text { Ratio }\end{array}$ & $\mathrm{r}$ & 0.23 & 0.16 & 0.22 & 0.21 & 0.13 \\
\cline { 2 - 7 } & $\mathrm{p}$ & $0.001^{* *}$ & $0.01^{*}$ & $0.001^{* *}$ & $0.003^{* *}$ & 0.06 \\
\hline $\begin{array}{c}\text { TC/ HDL-C C } \\
\text { Ratio }\end{array}$ & $\mathrm{r}$ & 0.20 & 0.27 & 0.23 & 0.18 & 0.21 \\
\cline { 2 - 7 } & $0.004^{* *}$ & $0.001^{* *}$ & $0.001^{* *}$ & $0.009^{* *}$ & $0.002^{* *}$ \\
\hline \multicolumn{7}{|c|}{ Table F } \\
\hline
\end{tabular}

3. Association of Lipid Profile and Hormonal Parameters (Table F).

In the present study significant correlation between lipid profile and hormonal parameters were shown in PCOS patients.

TSH had a significant positive relationship with TC, TG, LDL-C, VLDL-C, LDL-C/HDL-C ratio, and TC/HDL-C ratio whereas significant negative with HDL-C.LH had a significant positive relationship with TC, LDL-C, VLDL-C, LDL-C/HDL-C ratio, and $\mathrm{TC} / \mathrm{HDL}-\mathrm{C}$ ratio respectively and significant negative co-relation with HDL-C.FSH had a significant positive relationship with TC, TG, LDL-C, VLDL-C, LDLC/HDL-C ratio, and TC/HDL-C ratio whereas significant 
negative with HDL-C.PRL had a significant positive relationship with LDL-C, LDL-C/HDL-C ratio, and TC/HDL-C ratio whereas significant negative with HDL-C.LH/FSH ratio a significant positive relationship with TC and TC/HDL-C ratio and whereas significant negative with HDL-C in PCOS patients.

\section{DISCUSSION}

Polycystic Ovary syndrome is a common endocrinological disorder amongst women of reproductive age. The consequences of PCOS have reproductive repercussions as well as subsequent risk of metabolic syndrome, hypertension and cardiovascular disease.

\section{Anthropometric Measurements in PCOS Patients}

In our study $53 \%$ of the PCOS patients were found to be either overweight or obese (BMI $\left.>25 \mathrm{Kg} / \mathrm{m}^{2}\right)$ similar to the findings of Gomathi $\mathrm{K}^{14}$ et al, Cheng X, ${ }^{15}$ El-Hafeez $\mathrm{HA}^{16}$ et al and Rasool $\mathrm{SO}^{17}$ and contradictory to what was reported by Ahmadi et al. ${ }^{18}$ The marker of body fat content is body mass index (BMI) that increase the diagnostic power of identifying "at risk" groups. Yildiz BO, et al, ${ }^{19}$ Cheng X, et al ${ }^{15}$ reported that the prevalence of PCOS increased with the degree of obesity. Similarly, El-Hafeez HA et al. (2010) showed an increased BMI and waist circumference of PCOS women having insulin resistance. ${ }^{16}$ Saghafi-Asl et al. and Sousa RML et al. (2013) in their studies, documented significant positive correlations of anthropometric measures with lipid parameters, waist to height ratio (WHR) showed the strongest correlation with cholesterol (TC) $(r=0.37, p=0.004)$ and low density lipoprotein cholesterol (LDL-C) $(r=0.33, p=$ 0.011) amongst PCOS patients. ${ }^{20,21}$ Thathapudi et al. (2014) 22 documented that, the mean BMI, waist circumference, hip circumference, waist to hip ratio of PCOS subgroups were significantly higher $(\mathrm{p}<0.0001)$, than the controls or the sub groups with irregular mensuration, hyperandrogenism and polycystic ovaries. Rasool SO et al. (2015) ${ }^{17}$ and Shah AK et al. (2017) $^{23}$ studied the anthropometric measurements in women with PCOS and reported the presence of android obesity with regards to BMI and waist to hip ratio (WHR).

High body mass index and low serum HDL cholesterol are most frequently occurring components of the metabolic syndrome in PCOS. The present study has shown a significant increase in the mean levels of anthropometric parameters (BMI \& WHR) (Table A), and a positive correlation with PCOS subjects in accordance with the previous study shown by Kar S. 24

Waist-to-hip ratio (WHR) is a well-known factor associated with android type of obesity and in the presence of metabolic risk factors measurement of waist circumference can be more accurate to correlate excess abdominal fat than the total body fat. In the present study, $65 \%$ of the PCOS patients were found to have WHR $>85 \mathrm{~cm}$ (Table A) and is similar to the findings of Thathapudi $\mathrm{S}^{22}$ et al; but contrary to the findings of Rasool SO. ${ }^{17}$

Anthropometric characteristics (Especially BMI \& Hip circumference) are more important parameters related to lipid profile in PCOS patients. In our study, among anthropometric measures, BMI \& WHR showed significant positive correlations with lipid components (TC, TG, LDLC/HDL-C ratio, TC/HDL-C ratio, and significant negative correlation with HDL-C (Table D).

\section{Dyslipidaemia with PCOS}

In the present study dyslipidaemia associated with PCOS has been characterized by significant increase in the mean levels of all lipids (TC, TG, LDL-C, VLDL-C, LDL-C/HDL-C ratio, and TC/HDL-C ratio) and significant decrease in HDL-C levels in PCOS patients compared to the healthy subjects (Table B). These results are comparable to the results of Legro et al 25 and Diamanti Kandarakis E. ${ }^{26}$ LDL-C level was elevated in PCOS patients when compared to controls which has been reported by Legro et $\mathrm{al}^{25}$ as well. Regardless of the degree of obesity, a high proportion of women with PCOS are obese and are more likely to have central (Abdominal) distribution of body fat, which may be associated with insulin resistance and hyper androgenemia. ${ }^{26}$ Besides this Kalra et al found that insulin resistance is associated with dyslipidaemia in women with PCOS independent of obesity. ${ }^{27}$ Sarbhai V et al (2016) stated that clinical hormonal and metabolic alterations in women with PCOS were exacerbated by obesity, irrespective of clinical features like menstrual disturbances, infertility, hirsutism, acne and acanthiosis nigricans. ${ }^{28}$ However PCOS may intensify the adverse effects of obesity on insulin resistance. ${ }^{29} \mathrm{On}$ the other hand recent research by Zafar KS et $\mathrm{al}^{30}$ suggested that not all women with PCOS will develop dyslipidaemia and its complications but having PCOS enhances the chance for it Decreased HDL-C has been reported in more than two -thirds of PCOS subjects. 31 Our study has shown an increased level of FBS in PCOS patients indicating abnormal glucose metabolism similar to Rabelo Acevedo M et al.32 Deranged lipid profile in PCOS women and significant positive correlations of lipid profile with BMI and WHR has also been shown recently by Shah et al ${ }^{23}$ but this is contradictory to what has been reported by Kalra et al ${ }^{27}$ who did not find any such correlation.

\section{Hormonal Imbalances in PCOS}

In our study the mean values of all (TSH, LH, FSHand PRL) were significantly elevated in PCOS patients (Table C) indicating hormonal imbalances compared to controls as seen in other studies. ${ }^{23,33}$ Dipankar B et al ${ }^{34}$ noted $46.93 \%$ of PCOS had high LH/ FSH ratio. An inverse effect of LH and FSH on BMI and increased frequency of hirsutism in obese compared with lean PCOS women has been reported. 35 Cho LW et al. ${ }^{31}$ reported that LH/FSH ratio did not differ between the PCOS women and the non -affected group. Kumar A et al (2014) ${ }^{33}$ reported a significant increase in the levels of serum $\mathrm{LH}$; prolactin, TSH $(\mathrm{p}<0.001)$ and increased LH/FSH ratio $(>1.5)$ in women with PCOS compared with control women. In a recent case study, it was found that the levels of TSH, LH, FSH, prolactin and LH/FSH ratio were significantly raised in PCOS females $(p<0.0001)$ as compared to healthy females (Table E). Women with PCOS showed strong association between thyroid-stimulating hormone and insulin resistance independent of BMI and age and they had significantly altered endocrine and metabolic changes. ${ }^{36,37}$ Ganie MA et al. (2011) ${ }^{38}$ indicated that serum concentrations of triglyceride and TSH in patients with PCOS and subclinical hypothyroidism were higher than the controls. This is in concordance to the findings of Celik $\mathrm{C}$ et al. ${ }^{39}$ As per Cooper DS, and Benetti-Pinto CL et al subclinical hypothyroidism ( $\mathrm{SCH})$ is associated with higher low-density lipoprotein cholesterol and prolactin levels in young PCOS and no changes in other lipid parameters, insulin resistance or 
phenotypic manifestations. ${ }^{40,41}$ There are also reports documenting contrasting results such as Enzevaei A et al. (2014) who documented that there is no co-relation between subclinical hypothyroidism ( $\mathrm{SCH}$ ) and insulin resistance in PCOS women. 42 Hypothyroid disturbances and elevated thyroid stimulating hormone in PCOS women are associated with an adverse metabolic profile and the increased prevalence of subclinical hypothyroidism $(\mathrm{SCH})$ in women with PCOS might be the result of increased BMI, total cholesterol (TC)/high density lipoprotein cholesterol (HDL) ratio and lower free thyroxin and HDL cholesterol $(\mathrm{p}<0.05) .43,44.45$

Increased LH/FSH ratio has been shown to be the gold standard 46 and in our study LH/FSH ratio was found to be ( $>1.5$ ) compared to controls while Cho LW et al ${ }^{31}$ reported no change. In addition, raised serum LH levels has been reported to be associated with oligomenorrhoea in PCOS patients by Yosouf R et al. ${ }^{47}$ In our study compared to healthy controls TSH levels were significantly raised (Table F)and a strong positive correlation with anthropometric parameters and lipid profiles was seen. This is contrary to the levels found by Gomathi et al but corresponds to what has been reported by Kumar et $\mathrm{al}^{33}$ and Cooper DS. ${ }^{40}$ As Dittrich R et $\mathrm{al}^{37}$ women with PCOS showed strong association between TSH and insulin resistance independent of BMI and age.

\section{CONCLUSIONS}

Proper diagnosis and management of PCOS is essential as PCOS has many potential metabolic risks if not managed appropriately. ${ }^{48}$ Our study showed derangement in hormonal \& biochemical status of PCOS patients, high prevalence of hypothyroidism, and significant correlation of anthropometric parameters (especially BMI \& WHR) with lipid profile. Bargiota $A$. et al stated that even though treatment should be individualized, it should also focus on all metabolic consequences and on decreasing future complications. ${ }^{49}$ This reiterates the importance of early treatment of obesity, timely identification of hypothyroidism and biochemical derangement to prevent complications in the future. However more extensive research and understanding of the pathophysiology of PCOS will improve treatment success and overall management of patients. 50

\section{ACKNOWLEDGEMENT}

The authors are grateful to Prof Busi Karunanand for his continuous guidance, support and supervision in this study.

\section{REFERENCES}

[1] Umland EM, Weinstein LC, Buchanan EM. Menstruation-related disorders. In: DiPiro JT, Talbert RL, Yee GC, et al, eds. Pharmacotherapy: a Pathophysiologic Approach. 8th edn. New York: McGraw-Hill 2011: p. 1393.

[2] Diamanti-Kandarakis E, Kandarakis H, Legro RS. The role of genes and environment in the etiology of PCOS. Endocrine 2006;30(1):19-26.

[3] Shannon M, Wang Y. Polycystic ovary syndrome: a common but often unrecognized condition. J Midwifery Womens Health 2012;57(3):221-30.

[4] Norman RJ, Dewailly D, Legro RS, et al. Polycystic ovary syndrome. Lancet 2007;370(9588):685-97.
[5] Broekmans FJ, Knauff EA, Valkenburg 0, et al. PCOS according to the Rotterdam consensus criteria: change in prevalence among WHO-II anovulation and association with metabolic factors. BJOG 2006;113(10):1210-7.

[6] Lo JC, Feigenbaum SL, Yang J, et al. Epidemiology and adverse cardiovascular risk profile of diagnosed polycystic ovary syndrome. J Clin Endocrinol Metab 2006;91(4):1357-63.

[7] Nidhi R, Padmalatha V, Nagarathna R, et al. Prevalence of polycystic ovarian syndrome in Indian adolescents. J Pediatr Adolesc Gynecol 2011;24(4):223-7.

[8] Nair MK, Pappachan P, Balakrishnan S, et al. Menstrual irregularity and poly cystic ovarian syndrome among adolescent girls: a 2 year follow-up study. Indian J Pediatr 2012;79 Suppl 1:S69-73.

[9] Ganie MA, Kalra S. Polycystic ovary syndrome - a metabolic malady, the mother of all lifestyle disorders in women - Can Indian health budget tackle it in future? Indian J Endocrinol Metab 2011;15(4):23941.

[10] Vaidya RA. Polycystic Ovarian Syndrome, a Public Health Issue: Indian perspective oration delivered at the national institute for research in reproductive health ICMR- on the occasion of NIRRH 24 th Foundation Day.

[11] Joshi B, Mukherjee S, Patil A, et al. A cross-sectional study of polycystic ovarian syndrome among adolescent and young girls in Mumbai, India. Indian J Endocrinol Metab 2014;18(3):317-24.

[12] Rotterdam ESHRE/ASRM-sponsored PCOS Consensus Workshop group. Revised 2003 consensus on diagnostic criteria and long-term health risks related to polycystic ovary syndrome (PCOS). Fertil Steril 2004;81(1):19-25.

[13] Rotterdam ESHRE/ASRM-sponsored PCOS Consensus Workshop group. Revised 2003 consensus on diagnostic criteria and long -term health risks related to polycystic ovary syndrome (PCOS). Hum Reprod 2004;19(1):41-7.

[14] Gomathi K, Shaafie IA, Mummigatti K, et al. Biochemical parameters in women with polycystic ovary syndrome in Ajman, UAE. NJOG 2012;6(2):7-10.

[15] Cheng X, Ni R, Mo Y, et al. Appropriate BMI levels for PCOS patients in Southern China. Hum Reprod 2010;25(5):1295-302.

[16] El-Hafez HAA, Elrahawy MM, El-Aziz SA, et al. Thyroid function and volume are associated with anthropetric measurements and insulin resistance in Egyptian women with polycystic ovary syndrome. J Diabetes Metab 2013;4:288.

[17] Rasool SO. Anthropometric measurements in PCOS \& Non PCO infertile patients. International Journal of Chemical, Environmental \& Biological Sciences 2015;3(1):14-6.

[18] Ahmadi A, Akbarzadeh M, Mohammedi F, et al. Anthropometric characteristics and dietary pattern of women with polycystic ovary syndrome. Indian J Endocrinol Metab 2013;17(4):672-6. 
[19] Yidiz B0, Knochenhauer ES, Azziz R. Impact of obesity on the rise for polycystic ovary syndrome. J Clin Endocrinol Metab 2008;93(1):162-8.

[20] Saghafi-Asl M, Pirouzpanah S, Ebrahimi-Mameghani $\mathrm{M}$, et al. Lipid profile in relation to anthropometric indices and insulin resistance in overweight women with polycystic ovary syndrome. Health Promot Perspect 2013;3(2):206-16.

[21] de Sousa RM, Chein MB, da Silva DS, et al. Metabolic profile in women of different body mass indices with polycystic ovary syndrome. Rev Bras Ginecol Obstet 2013;35(9):413-20.

[22] Thatapudi S, Kodati V, Erukkambattu J, et al. Anthropometric and biochemical characteristics of polycystic ovarian syndrome in south indian women using AES-2006 criteria. Int J Endocrinol Metab 2014;12(1):e12470.

[23] Shah AK, Sarin M, Karunanand B, et al. Association of hormonal status with anthropometric and biochemical parameters in women with polycystic ovary syndrome. The Journal of Community Health Management 2017;4(1):30-4.

[24] Kar S. Anthropometric, clinical and metabolic comparisons of the four Rotterdam PCOS phenotypes: a prospective study of PCOS women. J Hum Reprod Sci 2013;6(3):194-200.

[25] Legro RS, Kunselman AR, Dunaif A. Prevalence and predictors of dyslipidemia in women with polycystic ovary syndrome. Am J Med 2001;111(8):607-13.

[26] Diamanti-Kandarakis E. Role of obesity and adiposity in polycystic ovary syndrome. Int J Obes (Lond) 2007;31 Suppl 2:S8-13.

[27] Kalra A, Nair S, Rai L. Association of obesity and insulin resistance with dyslipidemia in Indian women with polycystic ovarian syndrome. Indian J Med Sci 2006;60(11):447-53.

[28] Sarabhai V, Paul M. Impact of BMI on clinical, endocrine and metabolic profile of Indian PCOS women, GJRA-Gujarat. Journal for Research Analysis 2016:5(4)166-9.

[29] Behboudi-Gandevani S, Tehrani FR, Dovom MR, et al. Insulin resistance in obesity and polycystic ovary syndrome: systematic review and meta-analysis of observational studies. Gynecol Endocrinol 2016:32(5):343-53.

[30] Zafar KS, Pious T, Singh PS, et al. Prevalence of metabolic syndrome in a rural population- a cross sectional study from Western Uttar Pradesh, India. International Journal of Research in Medical Sciences Int J Res Med Sci 2017;5(5):2223-8.

[31] Cho LW, Jayagopal V, Kilpatrick ES, et al. The LH/FSH ratio has little use in diagnosing polycystic ovarian syndrome. Ann Clin Biochem 2006;43(Pt 3):217-9.

[32] Rabelo-Acevedo M, Ramírez-Vick M. Association between the polycystic ovary syndrome and the metabolic syndrome in Puerto Rico. Puerto Rico Health Sciences Journal 2005;24(3):203-6.

[33] Kumar AN, Naidu JN, Satyanarayana U, et al. Metabolic and endocrine characteristics of Indian women with polycystic ovary syndrome. Int J Fertil Steril 2016;10(1):22-8.
[34] Dipankar B, Kumar MS, Satinath M, et al. Clinical correlation with biochemical status in polycystic ovary syndrome. J Obstet Gynecol India 2005;55(1):67-71.

[35] Kiddy DS, Sharp PS, White DM, et al Differences in clinical and endocrine features between obese and non-obese subjects with polycystic ovary syndrome: an analysis of 263 consecutive cases. Clin Endocrinol (Oxf) 1990;32(2):213-20.

[36] Mueller A, Schöfl C, Dittrich R, et al. Thyroidstimulating hormone is associated with insulin resistance independently of body mass index and age in women with polycystic ovary syndrome. Hum Reprod 2009;24(11):2924-30.

[37] Dittrich R, Kajaia N, Cupisti S, et al. Association of thyroid-stimulating hormone with insulin resistance and androgen parameters in women with PCOS. Reprod Biomed Online 2009;19(3):319-25.

[38] Ganie MA, Laway BA, Wani TA, et al. Association of subclinical hypothyroidism and phenotype, insulin resistance, and lipid parameters in young women with polycystic ovary syndrome. Fertil Steril 2011;95(6):2039-43.

[39] Celik C, Abali R, Tasdemir N, et al. Is subclinical hypothyroidism contributing dyslipidemia and insulin resistance in women with polycystic ovary syndrome? Gynecol Endocrinol 2012;28(8):615-8.

[40] Cooper DS. Clinical practice. Subclinical hypothyroidism. N Engl J Med 2001:345(4):260-5.

[41] Benetti-Pinto CL, Berini Piccolo VR, Garmes HM, et al. Subclinical hypothyroidism in young women with polycystic ovary syndrome: an analysis of clinical, hormonal, and metabolic parameters. Fertil Steril 2013;99(2):588-92.

[42] Enzevaei A, Salehpour S, Tohidi M, et al. Subclinical hypothyroidism and insulin resistance in polycystic ovary syndrome: is there a relationship? Iran J Reprod Med 2014;12(7):481-6.

[43] Trummer C, Schwetz V, Giuliani A. et al. Impact of elevated thyroid-stimulating hormone levels in polycystic ovary syndrome. Gynecol Endocrinol 2015;31(10):819-23.

[44] Hueston WJ, Pearson WS. Subclinical hypothyroidism and the risk of hypercholesterolemia. Ann Fam Med 2004;2(4):351-5.

[45] Yasar HY, Topaloglu 0, Demirpence M. et al. Subclinical hypothyroidism in patients with polycystic ovary syndrome. Endocrine Abstracts 2015;37:EP217.

[46] Taylor AE. Gonadotropin dysfunction in women with polycystic ovary syndrome. Fertil Steril 2006;86 Suppl 1:S12.

[47] Yosouf R, Khan M, Kounsar Z, et al. Polycystic ovarian syndrome: clinical correlation with biochemical status. Surgical Science 2012;3(5):245-48.

[48] ACOG Committee on Practice Bulletins-Gynecology. ACOG Practice Bulletin No 108: polycystic ovary syndrome. Obstet Gynecol 2009;114(4):936-49. 
[49] Bargiota A, Diamanti-Kandarakis E. The effects of old, new, and emerging medicines on metabolic aberrations in PCOS. Ther Adv Endocrinol Metab 2012;3(1):27-47.
[50] Badawy A, Elnashar A. Treatment options for polycystic ovary syndrome. Int J Womens Health 2011;3:25-35. 